\title{
Using Data to Move from Volume to Value \\ \%
}

\author{
INTRODUCTION \\ Jason M. Sutherland, $\mathrm{PHD}$ \\ Professor \\ Centre for Health Services and Policy Research \\ School of Population and Public Health \\ Program Head, Health Services and Outcomes \\ Centre for Health Evaluation \& Outcome Sciences \\ Scholar, Michael Smith Foundation for Health Research \\ University of British Columbia \\ Vancouver, BC
}

Across Canada's provinces and territories, healthcare policy discussions are often focused on the attributes of the health services delivery system that are most easily measured and monitored. The obvious examples are spending growth, number of beds and wait times. In turn, this orientation tends to induce a focus on the volume of healthcare rather than its value. Yet it is increasingly clear that value from healthcare improves when patients' health goals are prioritized and addressed in a cost-efficient and effective manner.

Currently, value from healthcare is not routinely measured, even though doing so would inform whether governments are spending the "right" amount on healthcare or allocating it effectively between and within sectors. The gap between what information is measured now vis-à-vis what information is needed causes many to wonder if provincial and territorial delivery systems are reaching their potential.

The goal of healthcare is to improve or maintain quality of life, extend survival or relieve symptoms. In spite of its importance, measuring health is not done well; measuring quality of life that is important to patients and their caregivers is complex, measuring health or symptoms is not standardized and the costs of treatments are often unknown.

Not identifying or measuring what matters to patients, however, is a missed opportunity to align resources with patients' and caregivers' needs and, at the delivery system level, allocate 
resources to sectors or providers that provide the most value to patients.

Value-based healthcare is an approach to measuring value through patients' outcomes and spending to achieve those outcomes. The objective is to generate information to measure the effect of spending on health. Popularized in the United States (Porter 2010; Porter and Teisberg 2006) and spreading globally, the concept of value from healthcare is framed from the patient's perspective, built on the long history of measuring patients' outcomes relative to the cost of achieving the outcomes (Kaplan and Anderson 1990).

Internationally, incremental steps have begun to measure value from healthcare. Efforts include published frameworks for measuring value (Dubois and Westrich 2019; Garrison Jr et al. 2018; Willke et al. 2018), calls for collection of patients' outcomes (Gutacker and Street 2019; Porter et al. 2012), sector-based initiatives to improve value (Casalino and Khullar 2019) and movements to standardize outcome measures, such as the efforts of the International Consortium on Health Outcomes Measurement.

\section{What Should We Measure?}

The objective of this issue of Healthcare Papers is to draw attention to what data are needed, and when, to support innovations that will improve the value of healthcare across Canada. Through a series of linked commentaries, the authors propose concrete steps for providers, organizations and provincial governments to collect the "right" data and how the information it generates can pinpoint opportunities to improve on the delivery system's benefits to patients, providers and governments.

Significant progress on measuring value has been slow because consensus regarding what and when to measure has proven elusive, and results tend to be condition or symptom specific. As no notable progress has been made between provinces or territories, new ideas are needed on how to measure value from healthcare that are appropriate to the contexts of provinces' delivery systems.

In a single-payer delivery system, such as Canadian provinces, it is possible that value from healthcare is largely defined through political lenses and not through empirical evidence. However, the motivation that compels provinces to act on standardizing, measuring and valuing healthcare may come from the patient and caregiver engagement movement.

To this end, readers will find consistency of themes among the authors' commentaries regarding modernization of provinces' health data infrastructure. Each commentary recommends that patients' self-reported health and symptoms should be collected. Two commentaries provide a framework for determining what should be measured, when it should be measured and for how long (Horne and Manion 2019; Raveendran et al. 2019).

One commentary provides the lessons learned by detailing how self-reported health information is included in health system performance measurement (Jamieson Gilmore et al. 2019).

Concrete steps are proposed by a number of authors to address legislative or regulatory barriers to collecting self-reported information (Kuluski and Guilcher 2019) and identifying entities that could collect and report these new data (Horne and Manion 2019). Commentary authors also explain that to improve value from healthcare, expanded collection of cost information is called for (McCaughey et al. 2019) and that for the maximal benefits of measuring self-reported health to be realized, linkage to electronic medical records and administrative data is needed (Raveendran et al. 2019; Wong et al. 2019). 


\section{In This Issue}

This is the second of a four-part series devoted to value from healthcare; the first issue presented the concept of value from healthcare from the perspective of Canadian and international authors. Through a number of commentaries, the third issue will describe contemporaneous provincial and regional initiatives to improve value from healthcare. The final issue will have leading authors' thoughts regarding the future of value-based initiatives in Canada - pointing out where regulatory changes are needed and how to improve value from new information and articulate the type of research needed to complement province's activities in improving value from healthcare.

The commentary by McCaughey et al. (2019) advocates for the measurement of cost across the continuum of care and standardized collection of patient health outcomes. Leveraging cost and patient outcome data, the commentary proposes the following: conceptualization of value in the context to which it is being applied, operationalization by defining measures and accountabilities and implementation of process to improve outcomes. The commentary concludes with a call for action on behalf of the various levels of government and provider organizations and spells out specific roles for federal and provincial governments.

In their commentary, Kuluski and Guilcher (2019) explain that improving value is not sufficiently progressive and propose person-centred learning health systems (PC-LHSs). The authors' conceptualization of the PC-LHS first recognizes the importance of patients and caregivers beyond their condition and empowers patients and caregivers to express treatment preferences and participate in decision making that affects them. To support their contention, the authors assert that radically different streams of data are needed and that health and social care data need to be integrated. The PC-LHS would use this new linked information to be incorporated into quality improvement cycles. The authors conclude by describing features most likely to support the PC-LHS, including team-based care, co-location of services, data infrastructure that spans settings and different models of remuneration.

Raveendran et al. (2019) focus on pediatric surgery to highlight gaps in data and measurement, although the issues raised are relevant to many conditions and programs of care. The authors argue that current outcome indicators, such as surgical site infections, are less relevant to pediatric surgery because these are setting specific, relatively infrequent and insensitive to measuring value from interventions. The authors propose significant enhancements to data collection, including routinized collection of patient-reported outcome and experience measures (PROMs and PREMs) for measuring aspects of health likely to affect children's health and their families, including pain, anxiety, long-term health status and level of shared decision making. The authors conclude with a call for collection of PROMs and PREMs beyond the perioperative period and applying the data to improve quality and value through the structure of perioperative surgical homes.

In a complementary commentary by Wong et al. (2019), the authors take the perspective of primary care for arguing for population-based linkage of electronic medical record information, administrative data and patient-reported data. Stating that the performance of health systems is based on the strength of primary care, the authors articulate the need for PROMs and PREMs to measure value from primary care. The authors then highlight important issues that need to be resolved, such as language barriers associated with patient-based measurement and measurement of patients' contextual health and social environments, such as local health 
system priorities and availability of social care programs. The authors conclude by explaining that the key to effectively measuring value from healthcare is expanding patient-based data collection and linking it with other sources of information.

Taking a system lens, Horne and Manion (2019) propose a convener of value-based initiatives whose activities would include the following: maintaining an inventory of value-based initiatives, establishing provincial and national priorities for improving value in healthcare and leading policy development. Citing a gap in provincial leadership, the commentary looks to pan-Canadian health organizations with capacity, scale and experience to develop standards for interoperability between electronic medical records and embedding patient-reported outcomes into the processes of care. Finally, the authors articulate the need for an independent evaluator while noting that legal and regulatory frameworks need to be changed to enable information sharing, support benchmarking and identify new opportunities for co-investment between the government and the industry.

Providing an international perspective, a team of Italian researchers (Jamieson Gilmore

\section{References}

Casalino, L.P. and D. Khullar. 2019. Value-Based Purchasing and Physician Professionalism.

JAMA 322(17): 1647-48. doi:10.1001/ jama.2019.14990.

Dubois, R.W. and K. Westrich. 2019. As Value Assessment Frameworks Evolve, Are They Finally Ready for Prime Time? Value Health 22: 977-80. doi:10.1016/j.jval.2019.06.002.

Garrison Jr, L.P., P.J. Neumann, R.J. Willke, A. Basu, P.M. Danzon, J.A. Doshi, et al. 2018. A Health Economics Approach to US Value Assessment Frameworks - Summary and Recommendations of the ISPOR Special Task Force Report [7]. Value Health 21(2): 161-65. doi:10.1016/j.jval.2017.12.009. et al. 2019) highlight a regional process used to develop a multifactorial health systems performance assessment. The assessment framework, explicitly designed to include the patient voice, incorporates PROMs and PREMs, collected in a standardized format and comparable over time. The innovation described in this commentary provides inspiration for demonstrating how patientreported information can be reflected within routine health system performance and local quality improvement initiatives.

The issue of what health information should be collected is a complex challenge; provinces and territories have made limited gains collecting data for measuring value. Patients and governments will both benefit from improving value through better health outcomes and effective alignment of human and financial resources with what matters to patients. Canada provides a unique laboratory, with its separate delivery systems, to innovate in collecting self-reported health and health system cost information. Identifying modern sources of data for measuring value from healthcare is worth further development; this series of commentaries on health data needs is a first step to engaging in the deliberations.

Gutacker, N. and A. Street. 2019. Calls for

Routine Collection of Patient-Reported

Outcome Measures Are Getting Louder.

Journal of Health Services Research and Policy 24(1): 1-2. doi:10.1177/1355819618812239.

Horne, F. and R. Manion. 2019. A Made-in-Canada Approach to Value-Based Healthcare. Healthcare Papers 18(4): 10-19. doi:10.12927/hcpap.2019.26033.

Jamieson Gilmore, K.J., F. Pennucci, S. De Rosis and C. Passino. 2019. Value in Healthcare and the Role of the Patient Voice. Healthcare Papers 18(4): 28-35. doi:10.12927/hcpap.2019.26031.

Kaplan, R.M. and J.P. Anderson. 1990. The General Health Policy Model: An Integrated Approach. In B. Spiker, ed., Quality of Life Assessments in Clinical Trials (pp. 131-149). New York, NY: Raven Press. 
Kuluski, K. and S.T. Guilcher. 2019. Toward a Person-Centred Learning Health System: Understanding Value from the Perspectives of Patients and Caregivers. Healthcare Papers 18(4): 36-47. doi:10.12927/hcpap.2019.26030.

McCaughey, D., G. McGhan, S. Bele, N. Sharma and N.C. Ludlow. 2019. The Quest for Value in Canadian Healthcare: The Applied Value in Healthcare Framework. Healthcare Papers 18(4): 48-57. doi:10.12927/hcpap.2019.26029.

Porter, M., S. Larsson and M. Ingvar. 2012, October 31. A New Initiative to Put Outcomes Measurement at the Center of Health Reform. Health Affairs. Retrieved October 31, 2019. <https://www. healthaffairs.org/do/10.1377/hblog20121031.024785/ full/>.

Porter, M.E. 2010. What Is Value in Health Care? New England Journal of Medicine 363: 2477-81. doi:10.1056/NEJMp1011024.
Porter, M.E. and E.O. Teisberg. 2006. Redefining Health Care: Creating Value-Based Competition on Results. Cambridge, MA: Havard Business School Press.

Raveendran, L., M. Koyle and M. Brindle. 2019. Developing a Value-Based Approach to Outcome Reporting in Pediatric Surgery. Healthcare Papers 18(4): 20-27. doi:10.12927/hcpap.2019.26032.

Willke, R.J., P.J. Neumann, L.P. Garrison Jr and S.D. Ramsey. 2018. Review of Recent US Value Frameworks - A Health Economics Approach: An ISPOR Special Task Force Report [6]. Value Health 21(2): 155-60. doi:10.1016/j.jval.2017.12.011.

Wong, S.T., S. Johnston, F. Burge and K. McGrail. 2019. Value in Primary Healthcare - Measuring What Matters? Healthcare Papers 18(4): 58-67. doi:10.12927/hcpap.2019.26028.

\section{Longwoods Publishing Corporation}

\section{Announcement}

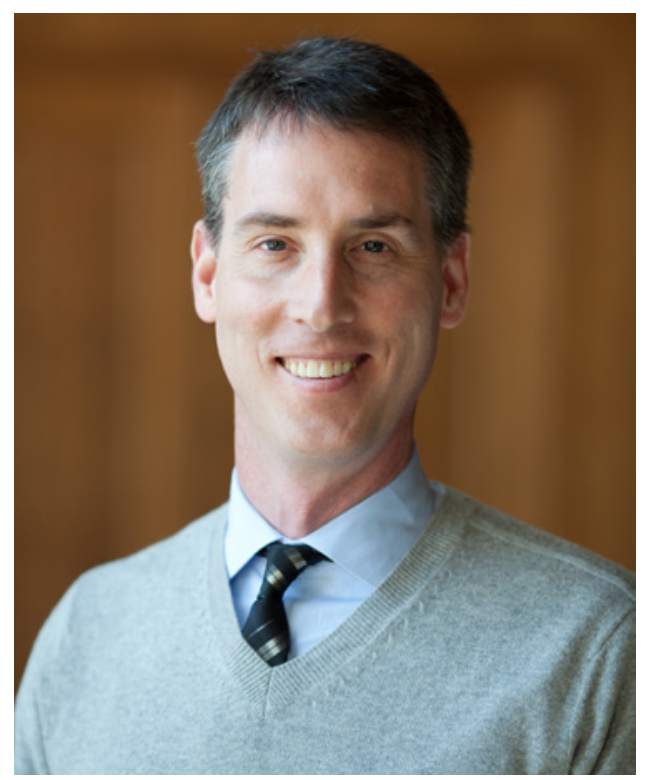

It is with great pleasure that Longwoods Publishing Corporation announces that Jason M. Sutherland, Professor, Centre for Health Services and Policy Research, University of British Columbia, has accepted the appointment as Editor-in-Chief of our journal, Healthcare Policy.

Prof. Sutherland succeeds Prof. Jennifer Zelmer who has led the journal for more than 10 years, building its reputation and strengthening its readership.

We encourage you to communicate to Prof. Zelmer, President and CEO at The Canadian Foundation for Healthcare Improvement, your thanks and gratitude for her successes with the journal.

Please also join Longwoods in welcoming Jason to the role of Editor-in-Chief. We look forward to his leadership and implementing his renewed vision for the journal.

Thank you,

\section{POLICY}

Politiques de Santé

HEALTHCAREPOLICY.NET

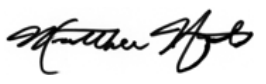

MATTHEW HART, CEO, LONGWOODS PUBLISHING 